TSL/ISV-2001-0249

June 2001

\title{
Hard colour singlet exchange at the Tevatron from the BFKL equation'
}

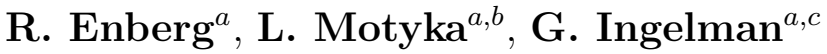 \\ ${ }^{a}$ High Energy Physics, Uppsala University, Uppsala, Sweden \\ ${ }^{b}$ Institute of Physics, Jagellonian University, Cracow, Poland \\ ${ }^{c}$ DESY, Hamburg, Germany
}

\begin{abstract}
We study rapidity gaps between jets in $p \bar{p}$ collisions at the Tevatron by a novel solution of the nonforward BFKL equation including nonleading effects through the consistency constraint and running coupling. Results differ from earlier calculations based on the asymptotic Mueller-Tang formula, but agree with D0 data when including full event simulation using PYTHIA to model the gap survival probability.
\end{abstract}

Mueller and Tang [G] (MT) proposed that events with two forward-backward high- $E_{T}$ jets with a large rapidity gap (of size $y$ ) between them could provide a test of the nonforward BFKL equation, in the form of elastic parton-parton scattering by hard $(|t| \gg 0)$ colour singlet exchange. However, the predictions obtained for the $t$ and $y$ dependence are not confirmed by $\mathrm{D} \varnothing[2]$ and $\mathrm{CDF}[3]$ data. To crudely reproduce the $t$-shape of the $\mathrm{D} \varnothing$ data the running of $\alpha_{s}(-t)$ had to be disregarded [ [4 and the $y$ dependence measured by CDF remains unexplained. The MT approximation is valid only for asymptotically large energies and neglects some contributions which may be relevant for the present experimental conditions. Here, we show that a more complete solution of the BFKL equation gives a good description of the data.

The cross section $d \hat{\sigma} / d t$ for elastic parton-parton scattering via BFKL pomeron exchange is dominated by the imaginary part of the amplitude

$$
\operatorname{Im} A(y, t)=\int \frac{d^{2} \mathbf{k}}{\pi} \frac{\Phi_{0}(\mathbf{k}, \mathbf{Q}) \Phi(y, \mathbf{k}, \mathbf{Q})}{\left[(\mathbf{k}+\mathbf{Q} / 2)^{2}+s_{0}\right]\left[(\mathbf{k}-\mathbf{Q} / 2)^{2}+s_{0}\right]},
$$

\footnotetext{
${ }^{1}$ Talk presented by RE at the 9th International Workshop on Deep Inelastic Scattering, Bologna, April 2001, to appear in the proceedings. E-mail: enberg@tsl.uu.se.
} 

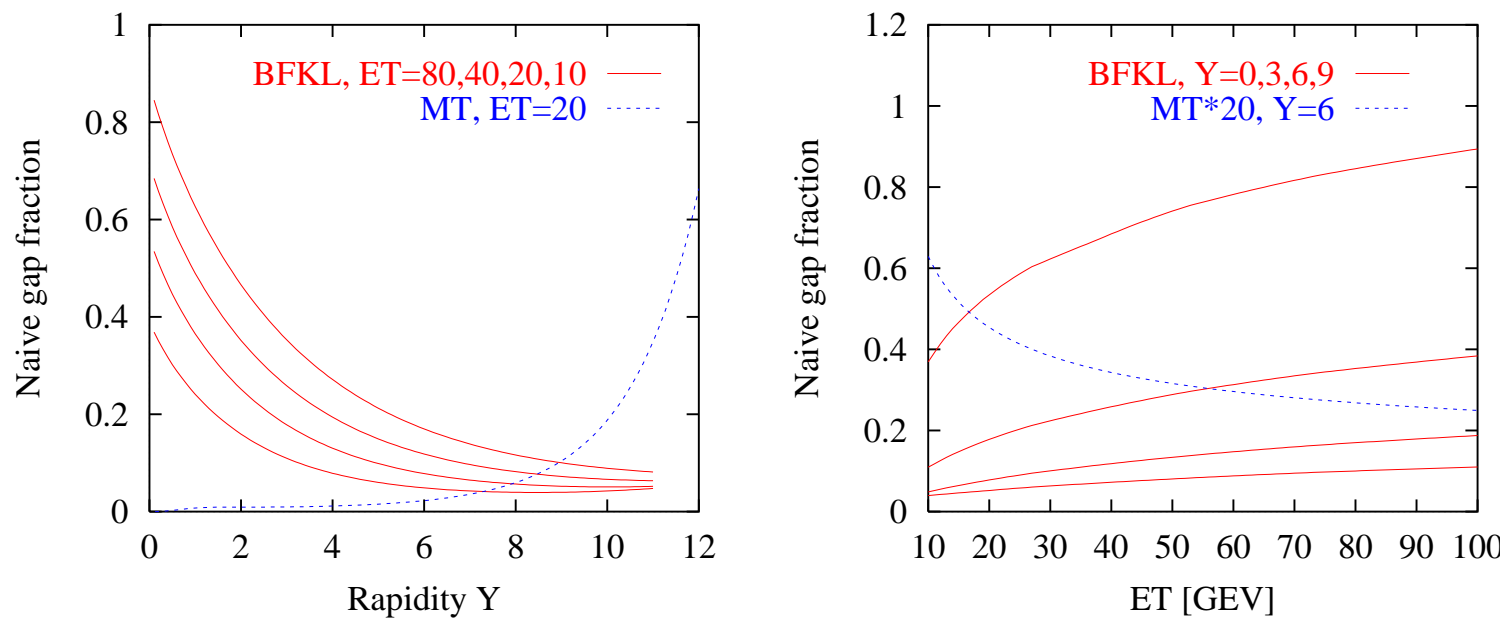

Figure 1: Parton level gap fractions (relative to LO QCD one-gluon exchange) in quarkquark scattering from our BFKL solution and the asymptotic MT approximation with the running coupling $\alpha_{s}(-t)$ in the prefactor, versus gap size $y$ (for the given $E_{T}$-values) and versus $E_{T}$ (for the given $y$-values). In the right-hand plot, the MT curve is multiplied by a factor of 20 .

where $\mathbf{Q} / 2 \pm \mathbf{k}$ denote the transverse momenta of the exchanged gluons and $t=-Q^{2}$. The scale $s_{0}$ is a physical infrared cut-off that reflects the confining properties of the QCD vacuum which suppresses the propagation of gluons with small virtualities. We set $s_{0}$ to $1 \mathrm{GeV}^{2}$, but note that the particular value affects mostly the normalisation and not the $y$ and $t$ dependence.

The impact factor $\Phi_{0}(\mathbf{k}, \mathbf{Q})$ is an effective vertex between the pomeron, the proton and the final state containing the high $E_{T}$ parton(s). At large $|t|$ and $y$, the pomeron predominantly couples to individual pointlike partons [5], corresponding to

$$
\Phi_{0}(\mathbf{k}, \mathbf{Q}) \sim \sqrt{\alpha_{s}\left((\mathbf{Q} / 2+\mathbf{k})^{2}+s_{0}\right) \alpha_{s}\left((\mathbf{Q} / 2-\mathbf{k})^{2}+s_{0}\right)} .
$$

We assume that this is a good approximation also in the nonasymptotic region. $\Phi(y, \mathbf{k}, \mathbf{Q})$ is the gluon distribution including full $y$ evolution which is found by solving the nonforward BFKL equation [6] with $\Phi_{0}(\mathbf{k}, \mathbf{Q})$ as the input. The nonleading corrections to the BFKL kernel are taken into account in an approximate way by including the consistency constraint [7] and using a running coupling where the scale $\mu^{2}=k^{2}+Q^{2} / 4$ is evaluated locally in the gluon ladder.

The BFKL equation is solved numerically by expanding it in a truncated Fourier series and discretising the system by the Tchebyshev interpolation method [8]. The resulting theoretical gap fraction is shown in fig. 1 together with the result of the MT formula which has a completely different behaviour. This is because MT corresponds to the asymptotically leading part of the scattering amplitude, which is not sensitive to the low scale details of the hadron structure and the QCD vacuum. However, the amplitude also contains a nonasymptotic part which depends on the physical cut-off scale and which dominates at 

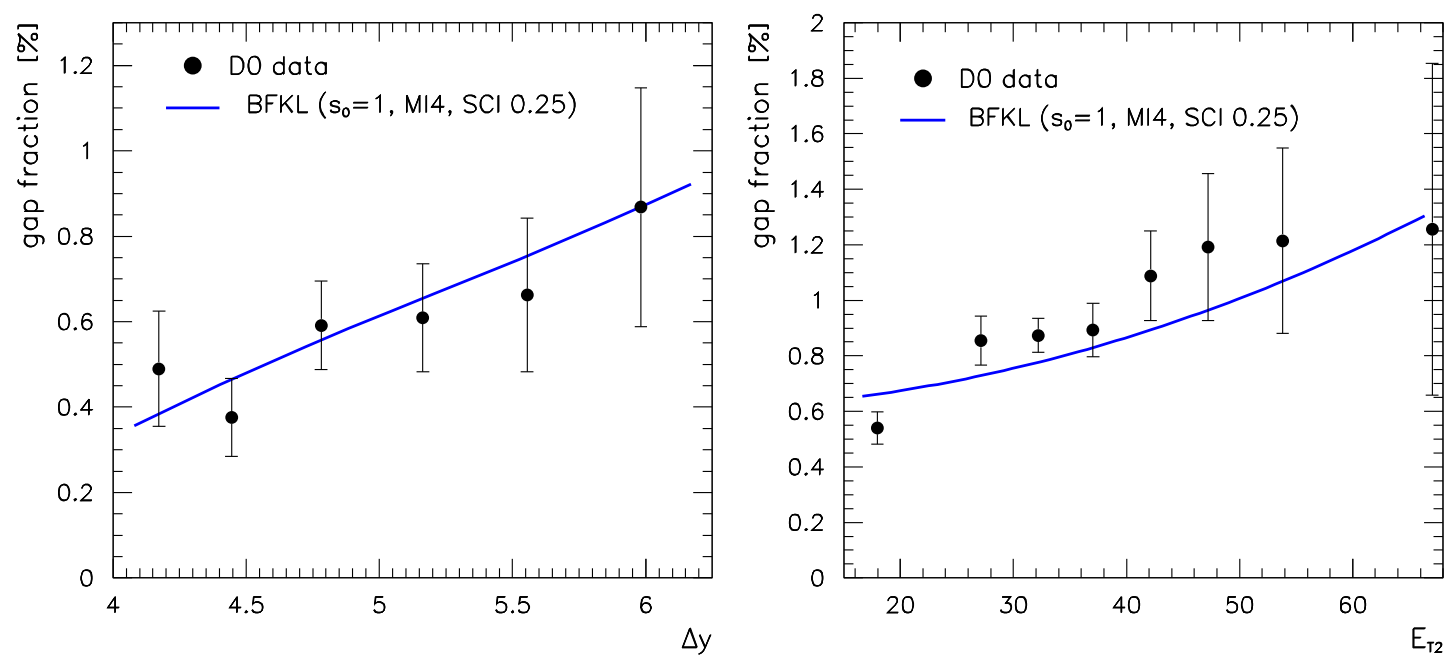

Figure 2: Fraction of jet-gap-jet events over all jet events versus jet separation $\Delta \eta$ for $15<E_{T 2}<25 \mathrm{GeV}$ and $E_{T}$ of the lowest energy jet; D $\varnothing$ data [2] compared to our BFKL-based model implemented in PYTHIA relative to all LO QCD $2 \rightarrow 2$ processes.

small $y$. This part contains a $\log \left(Q^{2} / s_{0}\right)$, visible at the Born level, which gets suppressed when $y$ increases. This is due to gluon reggeisation [5], i.e., the negative virtual correction terms in the BFKL kernel. The numerical value of $\log \left(Q^{2} / s_{0}\right)$ is large and the BFKL evolution is not rapid enough to reach the high $y$ asymptotic limit in the currently accessible domain of $y$. We stress, that this $\log \left(Q^{2} / s_{0}\right)$ in not an infrared artifact, since it would also appear if we considered the analogous process of heavy onia scattering, where $s_{0}$ $\left(\Lambda_{Q C D} \ll s_{0} \ll Q^{2}\right)$ would be a hard scale corresponding to the heavy onium size.

In order to compare with data, the parton level cross section discussed above must be convoluted with QCD evolved parton distributions of the incoming hadrons and the transition to final state hadrons be made. The latter is particularly important when considering rapidity gaps, which are defined in terms of a region without particles. One must then consider all additional activity in an event, such as higher order parton emissions, multiple parton scattering and hadronisation. A simple way of doing this is to multiply the cross section with an estimated gap survival probability. However, this probability cannot be independent of the subprocess, with its associated colour string topology, or of the kinematics in the event. For example, gluon-gluon scattering and a larger hard scale resulting in more parton radiation is expected to give a smaller gap survival probability.

The only way to handle these complex processes is via Monte Carlo event simulation. We have therefore implemented our cross section for elastic parton-parton scattering via colour singlet exchange as a new hard subprocess in the event generator PYTHIA [9]. Thus, higher order parton emissions are included through conventional parton showers and the underlying event is modelled with multiple interactions (using the best MI model $\operatorname{MSTP}(82)=4)$. To be consistent with our description [10] of rapidity gaps between a hard system $(W$, jets, bottom, $J / \psi)$ and a leading proton observed at the Tevatron, we also include the soft colour interaction (SCI) model [11]. The combination of SCI, with colour 
exchange probability $P=0.25$, and MI used here to model the underlying event is consistent with our previous study [10]. Although SCI was introduced to explain the creation of forward rapidity gaps, it has here the opposite effect by rearranging strings to span across the gap produced at the parton level by the BFKL singlet exchange.

The gap fraction resulting from this complete model describes quite well the $D \varnothing$ data, as shown in fig. 2. The normalisation of the gap ratio is, however, uncertain for two reasons. The parameter $s_{0}$ affects the normalisation of the parton level cross section and the simulated survival probability depends on the SCI model parameter $P$. A more complete study, including CDF data, will be presented in a coming paper [12].

In conclusion, we have solved the nonforward BFKL equation for colour singlet exchange including nonleading corrections. The part dominating at Tevatron conditions is not the asymptotically dominating part contained in the Mueller-Tang formula, but a part governed by a large logarithm that disappears in the large- $y$ limit. Using PYTHIA with full event simulation we obtain a dynamically varying gap survival probability resulting in a good description of the Tevatron data on rapidity gaps between jets.

We thank J. Kwieciński for stimulating discussions and acknowledge the support from the Swedish Natural Science Research Council. LM is supported in part by the KBN grant number 5 P P03B 14420.

\section{References}

[1] A.H. Mueller and W.-K. Tang, Phys. Lett. B 284, 123 (1992).

[2] B. Abbott et al., Phys. Lett. B 440, 189 (1998).

[3] F. Abe et al., Phys. Rev. Lett. 80, 1156 (1998).

[4] B. Cox, J. Forshaw and L. Lönnblad, JHEP 10, 023 (1999).

[5] J. Bartels et al., Phys. Lett. B 348, 589 (1995).

[6] L.N. Lipatov, Sov. J. Nucl. Phys. 23, 338 (1976); E.A. Kuraev et al., Sov. Phys. JETP 44, 443 (1976), ibid. Sov. Phys. JETP 45, 199 (1977); I. Balitsky et al., Sov. J. Nucl. Phys. 28, 822 (1978); L.N. Lipatov, Sov. Phys. JETP 63, 904 (1986).

[7] J. Kwieciński et al., Z. Phys. C 71, 585 (1996);

B. Andersson et al., Z. Phys. C 71, 613 (1996).

[8] J. Kwieciński, L. Motyka, Phys. Lett. B 438, 203 (1998).

[9] T. Sjöstrand et al., Comput. Phys. Commun. 135, 238 (2001).

[10] R. Enberg, G. Ingelman, N. Tîmneanu, hep-ph/0106246.

[11] A. Edin et al., Phys. Lett. B 366, 371 (1996); Z. Phys. C 75, 57 (1997).

[12] R. Enberg et al., in preparation. 